\title{
(D)-instanton effects in magnetized brane worlds
}

\author{
Marco Billó ${ }^{1 *}$ \\ ${ }^{1}$ Dip. di Fisica Teorica, Università di Torino and I.N.FN., sez. di Torino \\ Via Pietro Giuria 1, 10125 Torino (ITALY)
}

Key words Strings, branes, instantons, holomorphy.
PACS 11.25 -w, 11.25.Uv, 11.15.-q

We consider systems of magnetized D9 branes on orbifolds supporting $\mathcal{N}=1$ gauge theories. In such realizations, the matter multiplets arise from "twisted" strings connecting different stacks of branes. The introduction of Euclidean 5-branes wrapped on the six-dimensional compact space leads to instanton effects. We examine the interplay between the annuli diagrams with an E5 boundary and the holomorphicity properties of the effective low-energy supergravity action which describes the system, including its instanton corrections. This talk is mostly based on [1].

To appear in the proceedings of the RTN workshop "ForcesUniverse", Valencia, October 1-5 2007

\section{Introduction}

A popular and promising scenario for embedding phenomenologically appealing gauge and matter content in String Theory entails considering systems of magnetized D9 branes in type IIB Superstring, compactified on suitable six-dimensional manifolds; the T-dual description in terms of intersecting D6 branes in type IIA can also be used [2]. Such constructions support, in the uncompactified $\mathbf{R}^{1,3}$ space, supersymmetric gauge theories with chiral matter and interesting phenomenology. In particular, replica families arise naturally from multiple intersections, and different coupling constants can be tuned for the various gauge group factors. The low energy theory describing such four-dimensional theories is provided by supergravity coupled to vector and matter multiplets, and it can be derived directly from string amplitudes. Having and explicit string description makes it possible to investigate whether novel stringy effects, both perturbative and non-perturbative, might contribute to the effective action.

In this respect, it is by now clear that Euclidean E5 branes wrapped on the same cycle occupied by one of the stacks of D9 branes, which are point-like in $\mathbf{R}^{1,3}$, correspond to instantonic configurations of the gauge theory living on the D9 stack.

This is entirely analogous to the well-known D3/D(-1) system: the ADHM construction is naturally reproduced by the open strings attached to the instantonic branes [3, 4], while the non-trivial instanton profile of the gauge field is produced by the emission of gauge field vertices from mixed disks [5].

E5 branes wrapped differently from the D9 branes are still point-like in $\mathbf{R}^{1,3}$ but do not correspond to ordinary instantons configurations [6]. Still they can, in certain cases, give important non-perturbative, stringy contributions to the effective action, .e.g., Majorana masses for neutrinos [7, 8], moduli stabilizing terms and others, with a growing literature which impossible to quote here extensively. These "exotic" instantonic branes are thus potentially crucial for string based phenomenology.

Our aim here is to clarify some aspects of the "stringy instanton calculus", i.e., of the computation of Euclidean branes contributions. We focus on ordinary instantons, but our considerations should be useful for exotic instantons as well. We choose a toroidal compactification where string theory is calculable and realize (locally) $\mathcal{N}=1$ gauge SQCD on a system of D9-branes. We discuss then the contribution of E5 branes to the superpotential, analyzing the rôle of annuli bounded by E5 and D9 branes in giving these terms suitable holomorphicity properties.

* Corresponding author E-mail: billo@to.infn.it, Phone: +0039011670 7213, Fax: +0039011670 7213 


\section{The set-up}

We take as internal space the orbifold

$$
\frac{\mathcal{T}_{2}^{(1)} \times \mathcal{T}_{2}^{(2)} \times \mathcal{T}_{2}^{(3)}}{\mathbb{Z}_{2} \times \mathbb{Z}_{2}},
$$

whose Kähler and complex structures, $T^{(i)}$ and $U^{(i)}$ respectively with $i=1,2,3$ referring to the three tori, parametrize the string frame metric and the $B$ field. We decompose the 10 -d string fields into $4 \mathrm{~d}$ and $6 \mathrm{~d}$ components as $X^{M} \rightarrow\left(X^{\mu}, Z^{i}\right)$ and $\psi^{M} \rightarrow\left(\psi^{\mu}, \Psi^{i}\right)$, with $Z^{i}=\sqrt{\frac{T_{2}^{(i)}}{2 U_{2}^{(i)}}}\left(X^{2 i+2}+U^{(i)} X^{2 i+3}\right)$; the spin field decomposition we write as $S^{\dot{\mathcal{A}}} \rightarrow\left(S_{\alpha} S_{---}, S_{\alpha} S_{-++}, \ldots, S^{\dot{\alpha}} S^{+++}, \ldots\right)$. The three non-trivial orbifold elements $h_{i}$ act as follow:

$$
\begin{aligned}
& h_{1}:\left(Z^{1}, Z^{2}, Z^{3}\right) \rightarrow\left(Z^{1},-Z^{2},-Z^{3}\right), \\
& h_{2}:\left(Z^{1}, Z^{2}, Z^{3}\right) \rightarrow\left(-Z^{1}, Z^{2},-Z^{3}\right), \\
& h_{3}:\left(Z^{1}, Z^{2}, Z^{3}\right) \rightarrow\left(-Z^{1},-Z^{2}, Z^{3}\right) .
\end{aligned}
$$

The $\mathcal{N}=1$ supergravity describing the low energy theory of the closed string fields in this background can be expressed in terms of the fields $s, t^{(i)}, u^{(i)}$, with [2]

$$
\begin{aligned}
& \operatorname{Im}(s) \equiv s_{2}=\frac{1}{4 \pi} \mathrm{e}^{-\phi_{10}} T_{2}^{(1)} T_{2}^{(2)} T_{2}^{(3)} \\
& \operatorname{Im}\left(t^{(i)}\right) \equiv t_{2}^{(i)}=\mathrm{e}^{-\phi_{10}} T_{2}^{(i)}, u^{(i)}=u_{1}^{(i)}+\mathrm{i} u_{2}^{(i)}=U^{(i)}
\end{aligned}
$$

whose bulk Kähler potential reads [10]

$$
K=-\log \left(s_{2}\right)-\sum_{i=1} \log \left(t_{2}^{(i)}\right)-\sum_{i=1} \log \left(u_{2}^{(i)}\right) .
$$

We place now in this background a stack of $N_{a}$ fractional D9 branes (which we will call "color branes" and denote as $9 a$ branes). The massless spectrum of $9 a / 9 a$ strings gives rise, in $\mathbf{R}^{1,3}$, to the $\mathcal{N}=1$ vector multiplet for the gauge group $\mathrm{U}\left(N_{a}\right)$. The gauge coupling is given at tree level by ${ }^{1}$

$$
\frac{1}{g_{a}^{2}}=\frac{1}{4 \pi} \mathrm{e}^{-\phi_{10}} T_{2}^{(1)} T_{2}^{(2)} T_{2}^{(3)}=s_{2} .
$$

We add a further stack of D9-branes ("flavor branes" 9b) with quantized magnetic fluxes $f_{b}^{(i)}=$ $m_{b}^{(i)} / n_{b}^{(i)}$ and in a different orbifold representation. (Bulk) supersymmetry requires $\nu_{b}^{(1)}-\nu_{b}^{(2)}-\nu_{b}^{(3)}=0$, where

$$
f_{b}^{(i)} / T_{2}^{(i)}=\tan \pi \nu_{b}^{(i)}, \text { with } 0 \leq \nu_{b}^{(i)}<1
$$

Open strings stretched between the two stacks of branes ( $9 a / 9 b$ strings) are twisted by the relative angles

$$
\nu_{b a}^{(i)}=\nu_{b}^{(i)}-\nu_{a}^{(i)}
$$

\footnotetext{
${ }^{1}$ It is however the Wilsonian coupling $1 / \tilde{g}_{a}^{2}$ which has to be the imaginary part of a chiral multiplet, and it can in principle be corrected by sigma-model anomalies [9], so that$$
\frac{1}{\tilde{g}_{a}^{2}}=s_{2}=\frac{1}{g_{a}^{2}}-\frac{\delta}{8 \pi^{2}} .
$$

Only a shift $\delta^{(0)}$ could however play a role in our later discussion, see [1] for further details. 
If $\nu_{b a}^{(1)}-\nu_{b a}^{(2)}-\nu_{b a}^{(3)}=0$, this sector is supersymmetric: massless modes fill up a chiral multiplet $q_{b a}$ in the anti-fundamental representation $\bar{N}_{a}$ of the color group. The degeneracy of this chiral multiplet is $N_{b}\left|I_{a b}\right|$, where $I_{a b}$ is the number of Landau levels for the $(a, b)$ "intersection"

$$
I_{a b}=\prod_{i=1}\left(m_{a}^{(i)} n_{b}^{(i)}-m_{b}^{(i)} n_{a}^{(i)}\right) .
$$

We can now introduce a third stack of $9 c$ branes such that we get a chiral multiplet $q_{a c}$ in the fundamental representation $N_{a}$ and that

$$
N_{b}\left|I_{a b}\right|=N_{c}\left|I_{a c}\right| \equiv N_{F}
$$

This gives a (local) realization of $\mathcal{N}=1 \mathrm{SQCD}$, since we have the same number $N_{F}$ of fundamental and anti-fundamental chiral multiplets, respectively denoted by $q_{f}$ and $\tilde{q}^{f}$. The kinetic terms of the scalars $q_{f}$ sitting in the chiral multiplets, as evaluated from disk amplitudes, take a canonical form. This is not the case in the supergravity Lagrangian, where fields $Q_{f}$ with different normalizations are employed whose kinetic terms take the form

$$
\sum_{f=1}^{N_{F}}\left\{K_{Q} D_{\mu} Q^{\dagger f} D^{\mu} Q_{f}+K_{\tilde{Q}} D_{\mu} \tilde{Q}^{f} D^{\mu} \tilde{Q}_{f}^{\dagger}\right\}
$$

The "string" and "supergravity" fields are thus related via the Kähler metrics:

$$
q=\sqrt{K_{Q}} Q, \tilde{q}=\sqrt{K_{\tilde{Q}}} \tilde{Q}
$$

\subsection{Non-perturbative sectors from E5 branes}

In the string realization of SQCD described above, we can introduce instantonic sectors of the $\mathrm{U}\left(N_{a}\right)$ gauge theory by adding stacks of $k$ E5 branes whose internal part coincides with the D9 $a$. Notice that these branes would represent exotic instantons for the gauge theories on the D9 branes of type $b$ and $c$. We have new types of open strings: $\mathrm{E} 5_{a} / \mathrm{E} 5_{a}$ (neutral sector), $\mathrm{D} 9_{a} / \mathrm{E} 5_{a}$ (charged sector), $\mathrm{D} 9_{b} / \mathrm{E} 5_{a}$ or $\mathrm{E} 5_{a} / \mathrm{D} 9_{c}$ (flavored sectors, twisted). The states of these strings carry no momentum in space-time. They represent moduli, not fields. We shall indicate them collectively by $\mathcal{M}_{k}$; their spectrum and properties are summarized in table 1. Notice that charged or neutral moduli can have Kaluza-Klein momentum in (some of) the internal tori.

Among the neutral moduli we have the center of mass position $x_{0}^{\mu}$ and its fermionic partner $\theta^{\alpha}$, related to the supersymmetries broken by the E5a branes, which correspond to the singlet part:

$$
a^{\mu}=x_{0}^{\mu} \mathbb{1}_{k \times k}+y_{c}^{\mu} T^{c} \quad, \quad M^{\alpha}=\theta^{\alpha} \mathbb{1}_{k \times k}+\zeta_{c}^{\alpha} T^{c} .
$$

In the flavored sectors one has fermionic zero-modes only: the $\mu_{f}^{\prime}$ from the $\mathrm{D} 9_{b} / \mathrm{E} 5_{a}$ sector and the $\tilde{\mu}^{\prime f}$ from the $\mathrm{E} 5_{a} / \mathrm{D} 9_{c}$ sector.

\section{The stringy instanton calculus}

In presence of Euclidean branes, the dominant contributions to correlators of gauge/matter fields arise from products of one-point functions, connected by the integration over the moduli. The effective action for the gauge/matter fields is thus obtained by the functional integral over the instanton moduli of the exponential of all diagrams with at least part of their boundary on the E5a branes, possibly with insertions of moduli and gauge/matter fields[11]-[14],[5, ,7]. In the semi-classical approximation, only disk diagrams and annuli (the latter with no insertions) are retained. 


\begin{tabular}{cc|cccc}
\multicolumn{2}{c}{ Sector } & ADHM & Meaning & Chan-Paton & Dimension \\
\hline $5_{a} / 5_{a}$ & $\mathrm{NS}$ & $a_{\mu}$ & centers & adj. U $(k)$ & (length) \\
& & $D_{c}$ & Lagrange mult. & $\vdots$ & (length) $^{-2}$ \\
& $\mathrm{R}$ & $M^{\alpha}$ & partners & $\vdots$ & (length) $^{\frac{1}{2}}$ \\
& & $\lambda_{\dot{\alpha}}$ & Lagrange mult. & $\vdots$ & (length) $^{-\frac{3}{2}}$ \\
\hline $9_{a} / 5_{a}$ & $\mathrm{NS}$ & $w_{\dot{\alpha}}$ & sizes & $N_{a} \times \bar{k}$ & (length) \\
$5_{a} / 9_{a}$ & & $\bar{w}_{\dot{\alpha}}$ & $\vdots$ & $k \times \bar{N}_{a}$ & $\vdots$ \\
$9_{a} / 5_{a}$ & $\mathrm{R}$ & $\mu$ & partners & $N_{a} \times \bar{k}$ & (length) $^{\frac{1}{2}}$ \\
$5_{a} / 9_{a}$ & & $\bar{\mu}$ & $\vdots$ & $k \times \bar{N}_{a}$ & $\vdots$ \\
\hline $9_{b} / 5_{a}$ & $\mathrm{R}$ & $\mu^{\prime}$ & flavored & $N_{F} \times \bar{k}$ & (length) $^{\frac{1}{2}}$ \\
$5_{a} / 9_{c}$ & & $\tilde{\mu}^{\prime}$ & $\vdots$ & $k \times \bar{N}_{F}$ & $\vdots$
\end{tabular}

Table 1 The spectrum of moduli, labeled in accordance with their rôle in the ADHM construction.

In the case at hand, taking into account the dependence from the scalars $q, \tilde{q}$, the instantonic disk diagrams account for the shifted moduli action

$$
\begin{aligned}
S_{\text {mod }}\left(q, \tilde{q} ; \mathcal{M}_{k}\right) & =\operatorname{tr}_{k}\left\{\mathrm{i} D_{c}\left(\bar{w}_{\dot{\alpha}}\left(\tau^{c}\right)_{\dot{\beta}}^{\dot{\alpha}} w^{\dot{\beta}}+\mathrm{i} \bar{\eta}_{\mu \nu}^{c}\left[a^{\mu}, a^{\nu}\right]\right)\right. \\
& \left.-\mathrm{i} \lambda^{\dot{\alpha}}\left(\bar{\mu} w_{\dot{\alpha}}+\bar{w}_{\dot{\alpha}} \mu+\left[a_{\mu}, M^{\alpha}\right] \sigma_{\alpha \dot{\alpha}}^{\mu}\right)\right\} \\
& +\operatorname{tr}_{k} \sum_{f}\left\{\bar{w}_{\dot{\alpha}}\left[q^{\dagger f} q_{f}+\tilde{q}^{f} \tilde{q}_{f}^{\dagger}\right] w^{\dot{\alpha}}-\frac{\mathrm{i}}{2} \bar{\mu} q^{\dagger f} \mu_{f}^{\prime}+\frac{\mathrm{i}}{2} \tilde{\mu}^{\prime f} \tilde{q}_{f}^{\dagger} \mu\right\} .
\end{aligned}
$$

There are other relevant diagrams which involve the superpartners of $q$ and $\tilde{q}$ and are related to the above by susy Ward identities. The complete result is obtained by letting

$$
q\left(x_{0}\right), \tilde{q}\left(x_{0}\right) \rightarrow q\left(x_{0}, \theta\right), \tilde{q}\left(x_{0}, \theta\right)
$$

in $S_{\bmod }\left(q, \tilde{q} ; \mathcal{M}_{k}\right)$. Using this ingredient, the effective action in the Higgs branch takes the form

$$
S_{k}=\mathcal{C}_{k} \mathrm{e}^{-\frac{8 \pi^{2}}{g_{a}^{2}} k} \mathrm{e}^{\mathcal{A}_{5 a}^{\prime}} \int d \mathcal{M}_{k} \mathrm{e}^{-S_{\bmod }\left(q, \tilde{q} ; \mathcal{M}_{k}\right)} .
$$

In $\mathcal{A}_{5 a}^{\prime}$ the contribution of zero-modes running in the loop is suppressed because they are already explicitly integrated over [7, 15, 16, 17]. $\mathcal{C}_{k}$ is a normalization factor, determined up to numerical constants by counting the dimensions, measured in units of $\alpha^{\prime}$, of the moduli $\mathcal{M}_{k}$ :

$$
\mathcal{C}_{k}=\left(\sqrt{\alpha^{\prime}}\right)^{-\left(3 N_{a}-N_{F}\right) k}\left(g_{a}\right)^{-2 N_{a} k} .
$$

Notice the appearance of the $\beta$-function coefficient. $b_{1}=N_{a}-N_{F}$.

In $S_{\text {mod }}\left(q, \tilde{q} ; \mathcal{M}_{k}\right)$, the superspace coordinates $x_{0}^{\mu}$ and $\theta^{\alpha}$ appear only within the superfields $q\left(x_{0}, \theta\right)$, $\tilde{q}\left(x_{0}, \theta\right), \ldots$ We can thus separate $x, \theta$ from the other moduli $\widehat{\mathcal{M}}_{k}$ writing

$$
S_{k}=\int d^{4} x_{0} d^{2} \theta W_{k}(q, \tilde{q})
$$

in terms of the effective superpotential

$$
W_{k}(q, \tilde{q})=\mathcal{C}_{k} \mathrm{e}^{-\frac{8 \pi^{2}}{g_{a}^{2}} k} \mathrm{e}^{\mathcal{A}_{5_{a}}^{\prime}} \int d \widehat{\mathcal{M}}_{k} \mathrm{e}^{-S_{\bmod }\left(q, \tilde{q} ; \widehat{\mathcal{M}}_{k}\right)} .
$$


This expression seems not to be holomorphic, as a superpotential should be. Indeed, $S_{\text {mod }}\left(q, \tilde{q} ; \widehat{\mathcal{M}}_{k}\right)$ explicitly depends on $q^{\dagger}$ and $\tilde{q}^{\dagger}$. This dependence disappears upon integrating over $\widehat{\mathcal{M}}_{k}$ as a consequence of the cohomology properties of the integration measure on the instanton moduli space [18, 19, 20]. However, we have to re-express the result in terms of the supergravity fields $Q$ and $\tilde{Q}$ via the rescalings in eq. 12 and this reintroduces non-holomorphicity. At the same time, we have to use the holomorphic dynamically generated scale $\Lambda_{\text {hol }}$, obtained by integrating the Wilsonian $\beta$-function of the $\mathcal{N}=1$ SQCD [21, 19]:

$$
\Lambda_{\mathrm{hol}}^{b_{1}}=\left(\sqrt{\alpha^{\prime}}\right)^{-b_{1}} \mathrm{e}^{-\frac{8 \pi^{2}}{\tilde{g}_{a}^{2}}},
$$

where the holomorphic coupling $\tilde{g}_{a}$ of 5 appears. Finally, $\mathcal{A}_{5_{a}}^{\prime}$ can introduce a non-holomorphic dependence on the complex and Kähler structure moduli of the compactification space. Our aim is to consider the interplay of all these observations; for this we need the explicit expression of the mixed annuli term $\mathcal{A}_{5_{a}}^{\prime}$, which we will discuss shortly. For the time being, let us consider the one-instanton sector, $k=1$. In this case, the integral over the moduli can be carried out explicitly. Balancing the fermionic zero-modes requires that $N_{F}=N_{a}-1$ and the end result is [19] (see also Refs. [16, 22]).

$$
W_{k=1}(q, \tilde{q})=\mathcal{C}_{k} \mathrm{e}^{-\frac{8 \pi^{2}}{g_{a}^{2}} k} \mathrm{e}^{\mathcal{A}_{5 a}^{\prime}} \frac{1}{\operatorname{det}(\tilde{q} q)} .
$$

This superpotential has basically the same form as the ADS/TVY superpotential [23, 24] except for the prefactors discussed above: we shall see how these factors precisely conspire to give an holomorphic expression in the supergravity variables $Q$ and $\tilde{Q}$.

\section{Instanton annuli and threshold corrections}

The amplitude $\mathcal{A}_{5_{a}}$ is a sum of cylinder amplitudes with a boundary on the E5a (both orientations) These

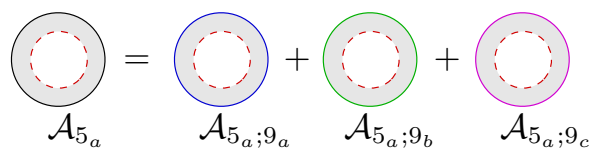

Fig. 1 Mixed annuli diagrams appearing in the stringy instanton calculus

amplitudes are both UV and IR divergent. The UV divergences (IR in the closed string channel) cancel if tadpole cancellation assumed. The IR divergence is regulated with a scale $\mu$. As noticed in [15, 16], there is a relation between these instantonic annuli and the running gauge coupling constant:

$$
\mathcal{A}_{5_{a}}=-\left.\frac{8 \pi^{2} k}{g_{a}^{2}(\mu)}\right|_{1-\text { loop }} .
$$

Indeed, in supersymmetric theories, mixed annuli compute the running coupling by expanding around the instanton background, as discussed in [17].

This relation is confirmed by the explicit computation of the annuli. Imposing the appropriate boundary conditions and the GSO projection one starts from

$$
\int_{0}^{\infty} \frac{d \tau}{2 \tau}\left[\operatorname{Tr}_{\mathrm{NS}}\left(P_{\mathrm{GSO}} P_{\text {orb. }} q^{L_{0}}\right)-\operatorname{Tr}_{\mathrm{R}}\left(P_{\mathrm{GSO}} P_{\text {orb. }} q^{L_{0}}\right)\right]
$$

For $\mathcal{A}_{5_{a} ; 9_{a}}$, KK copies of zero-modes on internal tori $\mathcal{T}_{2}^{(i)}$ give a non-holomorphic dependence on the Kähler and complex moduli (threshold correction) and one finds [25, 26]

$$
\mathcal{A}_{5_{a} ; 9_{a}}=-8 \pi^{2} k\left[\frac{3 N_{a}}{16 \pi^{2}} \log \left(\alpha^{\prime} \mu^{2}\right)+\frac{N_{a}}{16 \pi^{2}} \sum_{i} \log \left(U_{2}^{(i)} T_{2}^{(i)}\left(\eta\left(U^{(i)}\right)^{4}\right)\right],\right.
$$


For $\mathcal{A}_{5_{a} ; 9_{b}}$ and $\mathcal{A}_{5_{a} ; 9_{c}}^{\prime}$, the modes are twisted and the result depends on the angles $\nu_{b a}^{(i)}$ and $\nu_{a c}^{(i)}$ :

$$
\mathcal{A}_{5_{a} ; 9_{b}}+\mathcal{A}_{5_{a} ; 9_{c}}=8 \pi^{2} k\left(\frac{N_{F}}{16 \pi^{2}} \log \left(\alpha^{\prime} \mu^{2}\right)+\frac{N_{F}}{32 \pi^{2}} \log \left(\boldsymbol{\Gamma}_{b a} \boldsymbol{\Gamma}_{a c}\right)\right),
$$

where

$$
\boldsymbol{\Gamma}_{b a}=\frac{\Gamma\left(1-\nu_{b a}^{(1)}\right)}{\Gamma\left(\nu_{b a}^{(1)}\right)} \frac{\Gamma\left(\nu_{b a}^{(2)}\right)}{\Gamma\left(1-\nu_{b a}^{(2)}\right)} \frac{\Gamma\left(\nu_{b a}^{(3)}\right)}{\Gamma\left(1-\nu_{b a}^{(3)}\right)}
$$

and $\boldsymbol{\Gamma}_{a c}$ has an analogous expression.

Summing all types of annuli, we notice that the coefficient of the IR divergent term $\log \alpha^{\prime} \mu^{2}$ is proportional to the $\beta$-function coefficient of SQCD $b_{1}=3 N_{a}-N_{F}$.

\section{Holomorphicity properties}

As stressed before, the instanton-induced correlators involve the primed part $\mathcal{A}_{5 a}^{\prime}$ of the mixed annuli, deprived of the the contributions of the zero-modes running in the loop, which are responsible for the IR divergences. To isolate such contributions, we use the natural UV cut-off of the low-energy theory, the Planck mass

$$
M_{P}^{2}=\frac{1}{\alpha^{\prime}} \mathrm{e}^{-\phi_{10}} s_{2} .
$$

We write then

$$
\mathcal{A}_{5 a}=-k \frac{b_{1}}{2} \log \frac{\mu^{2}}{M_{P}^{2}}+\mathcal{A}_{5 a}^{\prime} .
$$

With some algebra, and recalling the definition of the supergravity variables, we find

$$
\mathcal{A}_{5_{a}}^{\prime}=-N_{a} \sum_{i=1}^{3} \log \left(\eta\left(u^{(i)}\right)^{2}\right)+N_{a} \log g_{a}^{2}+\frac{N_{a}-N_{F}}{2} K+\frac{N_{F}}{2} \log \left(\mathcal{Z}_{b a} \mathcal{Z}_{a c}\right)
$$

with (similarly for $\mathcal{Z}_{a c}$ )

$$
\mathcal{Z}_{b a}=\left(4 \pi s_{2}\right)^{-\frac{1}{4}}\left(t_{2}^{(1)} t_{2}^{(2)} t_{2}^{(3)}\right)^{-\frac{1}{4}}\left(u_{2}^{(1)} u_{2}^{(2)} u_{2}^{(3)}\right)^{-\frac{1}{2}}\left(\boldsymbol{\Gamma}_{b a}\right)^{\frac{1}{2}} .
$$

Let us focus now on the one instanton case $k=1$. When $N_{F}=N_{a}-1$ we found the effective superpotential of eq. 20]. Inserting the explicit form of the annuli, eq. 28], and rewriting the resulting expression in terms of the holomorphic scale $\Lambda_{\text {hol }}$ of eq. [19], we get

$$
W_{k=1}(q, \tilde{q})=\mathrm{e}^{K / 2} \prod_{i=1}^{3}\left(\eta\left(u^{(i)}\right)^{-2 N_{a}}\right) \Lambda_{\mathrm{hol}}^{2 N_{a}+1}\left(K_{b a} K_{a c}\right)^{\frac{N_{a}-1}{2}} \frac{1}{\operatorname{det}(\tilde{q} q)},
$$

where, taking into account a possible shift $\delta^{(0)}$ in the definition of the Wilsonian coupling, see eq. 5), we have introduced

$$
K_{a b}=\chi_{a b} \mathcal{Z}_{a b},
$$

with the multiplicative corrections $\chi_{a b}$ satisfying

$$
\delta^{(0)}+\frac{N_{F}}{2} \log \chi_{b_{a}} \chi_{a c}=0
$$


We can then make an holomorphic redefinition of the scale $\Lambda_{\text {hol }}$ into $\widehat{\Lambda}_{\text {hol }}$ and rescale the chiral multiplet to their supergravity counterparts. If we assume that the Kähler metrics for the chiral multiplets are given by

$$
K_{Q}=K_{b a}, K_{\tilde{Q}}=K_{a c}
$$

we finally obtain an expression which fits perfectly in the low energy Lagrangian:

$$
W_{k=1}(Q, \tilde{Q})=\mathrm{e}^{K / 2} \widehat{\Lambda}_{\mathrm{hol}}^{2 N_{a}+1} \frac{1}{\operatorname{det}(\tilde{Q} Q)}
$$

which, a part from the prefactor $\mathrm{e}^{\frac{K}{2}}$, is holomorphic in the variables of the Wilsonian scheme.

The holomorphicity properties of the instanton-induced superpotential suggest that the Kähler metric of chiral multiplets $Q$ arising from twisted $\mathrm{D} 9_{a} / \mathrm{D} 9_{b}$ strings is given, a part from the multiplicative corrections $\chi_{b a}$ of eq. 31, by

$$
K_{Q}=\left(4 \pi s_{2}\right)^{-\frac{1}{4}}\left(t_{2}^{(1)} t_{2}^{(2)} t_{2}^{(3)}\right)^{-\frac{1}{4}}\left(u_{2}^{(1)} u_{2}^{(2)} u_{2}^{(3)}\right)^{-\frac{1}{2}}\left(\boldsymbol{\Gamma}_{b a}\right)^{\frac{1}{2}},
$$

with

$$
\boldsymbol{\Gamma}_{b a}=\frac{\Gamma\left(1-\nu_{b a}^{(1)}\right)}{\Gamma\left(\nu_{b a}^{(1)}\right)} \frac{\Gamma\left(\nu_{b a}^{(2)}\right)}{\Gamma\left(1-\nu_{b a}^{(2)}\right)} \frac{\Gamma\left(\nu_{b a}^{(3)}\right)}{\Gamma\left(1-\nu_{b a}^{(3)}\right)} .
$$

This is very interesting because for twisted fields, the Kähler metric cannot be derived from compactification of the DBI action. The part depending on the twists, namely $\Gamma_{b a}$, is reproduced by a direct string computation [27, 28]. The prefactors, depending on the geometric moduli, are more difficult to get directly: the present suggestion is welcome.

This expression of the Kähler metric can be checked against the known results for Yukawa couplings of magnetized branes [29], finding perfect consistency.

\subsection{Relation with the perturbative approach and the DKL formula}

We have discussed the relation between the instantonic annuli and the running gauge coupling. In turn, there is a general relation of the 1-loop corrections [30, 31] to the Wilsonian gauge coupling $f$ :

$$
\frac{1}{g^{2}(\mu)}=\frac{1}{\tilde{g}^{2}} \frac{1}{8 \pi^{2}}\left[\frac{b}{2} \log \frac{\mu^{2}}{M_{P}^{2}}-f^{(1)}-\frac{c}{2} K+T(G) \log \frac{1}{\tilde{g}^{2}}-\sum_{r} n_{r} T(r) \log K_{r}\right],
$$

where $T_{A}$ are the generators of the gauge group, $n_{r}$ is the number of chiral multiplets in the representation $r$ and

$$
\begin{aligned}
& T(r) \delta_{A B}=\operatorname{Tr}_{r}\left(T_{A} T_{B}\right) \quad, \quad T(G)=T(\operatorname{adj}) \\
& b=3 T(G)-\sum_{r} n_{r} T(r) \quad, \quad c=T(G)-\sum_{r} n_{r} T(r) .
\end{aligned}
$$

This gives an interpretation for the non-holomorphic terms appearing in the running coupling based on perturbative considerations.

In the case of SQCD, one has $N_{F}$ chiral multiplets in the representations $N_{a}$ and $\bar{N}_{a}$. Matching the DKL formula with the 1-loop result for $1 / g_{A}^{2}(\mu)$ one can identify the Kähler metrics $K_{Q}$ and $K_{\tilde{Q}}$ of the chiral multiplets. This determination, based on the holomorphicity of perturbative contributions to the effective action, is in full agreement with the expression given before, derived from the holomorphicity of instanton contributions. 


\section{Remarks and conclusions}

The type of analysis reviewed here can be applied also in $\mathcal{N}=2$ toroidal models, where one can show that the instanton-induced superpotential is in fact holomorphic in the appropriate supergravity variables if one includes the mixed annuli in the stringy instanton calculus [16, 17]. In the present analysis, we have focused on the fact that, with respect to the "color" D $9_{a}$ branes, the $\mathrm{E} 5_{a}$ branes represent ordinary instantons. For the gauge theories on the $\mathrm{D} 9_{b}$ or the $\mathrm{D} 9_{c}$, they would be exotic instantons, whose status is not yet completely clear from the field theory viewpoint. The study of the mixed annuli and their relation to holomorphicity can thus be relevant for exotic, new stringy effects as well.

\section{References}

[1] M. Billo, M. Frau, I. Pesando, P. Di Vecchia, A. Lerda and R. Marotta, "Instanton effects in N=1 brane models and the Kahler metric of twisted matter," arXiv:0709.0245 [hep-th].

[2] For a review, see for instance R. Blumenhagen, B. Kors, D. Lust and S. Stieberger, Phys. Rept. 445 (2007) 1 arXiv:hep-th/0610327.

[3] E. Witten, Nucl. Phys. B 460 (1996) 335 [arXiv:hep-th/9510135].

[4] M. R. Douglas, arXiv:hep-th/9512077

[5] M. Billo, M. Frau, I. Pesando, F. Fucito, A. Lerda and A. Liccardo, JHEP 0302 (2003) 045 |arXiv:hepth/0211250].

[6] C. Beasley and E. Witten, JHEP 0602 (2006) 060 [arXiv:hep-th/0512039].

[7] R. Blumenhagen, M. Cvetic and T. Weigand, Nucl. Phys. B 771 (2007) 113 [arXiv:hep-th/0609191].

[8] L. E. Ibanez and A. M. Uranga, JHEP 0703 (2007) 052 [arXiv:hep-th/0609213].

[9] J. P. Derendinger, S. Ferrara, C. Kounnas and F. Zwirner, Nucl. Phys. B 372 (1992) 145.

[10] I. Antoniadis, C. Bachas, C. Fabre, H. Partouche and T. R. Taylor, Nucl. Phys. B 489 (1997) 160 |arXiv:hepth/9608012.

[11] J. Polchinski, Phys. Rev. D 50 (1994) 6041 [arXiv:hep-th/9407031].

[12] M. B. Green and M. Gutperle, Nucl. Phys. B 498 (1997) 195 |arXiv:hep-th/9701093].

[13] M. B. Green and M. Gutperle, JHEP 9801 (1998) 005 [arXiv:hep-th/9711107].

[14] M. B. Green and M. Gutperle, Phys. Rev. D 58 (1998) 046007 [arXiv:hep-th/9804123].

[15] S. A. Abel and M. D. Goodsell, JHEP 0710 (2007) 034 |arXiv:hep-th/0612110|.

[16] N. Akerblom, R. Blumenhagen, D. Lust, E. Plauschinn and M. Schmidt-Sommerfeld, JHEP 0704 (2007) 076 [arXiv:hep-th/0612132].

[17] M. Billo, M. Frau, I. Pesando, P. Di Vecchia, A. Lerda and R. Marotta, JHEP 0710 (2007) 091 arXiv:0708.3806 [hep-th]].

[18] T. J. Hollowood, JHEP 0203 (2002) 038 [arXiv:hep-th/0201075].

[19] N. Dorey, T. J. Hollowood, V. V. Khoze and M. P. Mattis, Phys. Rept. 371 (2002) 231 [arXiv:hep-th/0206063].

[20] M. Billo, M. Frau, F. Fucito and A. Lerda, JHEP 0611 (2006) 012 [arXiv:hep-th/0606013].

[21] V. A. Novikov, M. A. Shifman, A. I. Vainshtein and V. I. Zakharov, Nucl. Phys. B 229 (1983) 381.

[22] R. Argurio, M. Bertolini, G. Ferretti, A. Lerda and C. Petersson, JHEP 0706 (2007) 067 [arXiv:0704.0262 [hepth]].

[23] T. R. Taylor, G. Veneziano and S. Yankielowicz, Nucl. Phys. B 218 (1983) 493.

[24] I. Affleck, M. Dine and N. Seiberg, Nucl. Phys. B 241 (1984) 493.

[25] D. Lust and S. Stieberger, Fortsch. Phys. 55 (2007) 427 |arXiv:hep-th/0302221].

[26] N. Akerblom, R. Blumenhagen, D. Lust and M. Schmidt-Sommerfeld, Phys. Lett. B 652 (2007) 53 [arXiv:0705.2150 [hep-th]]

[27] D. Lust, P. Mayr, R. Richter and S. Stieberger, Nucl. Phys. B 696 (2004) 205 [arXiv:hep-th/0404134].

[28] M. Bertolini, M. Billo, A. Lerda, J. F. Morales and R. Russo, Nucl. Phys. B 743 (2006) 1 |arXiv:hep-th/0512067|.

[29] D. Cremades, L. E. Ibanez and F. Marchesano, JHEP 0307 (2003) 038 [arXiv:hep-th/0302105].

[30] L. J. Dixon, V. Kaplunovsky and J. Louis, Nucl. Phys. B 355 (1991) 649.

[31] V. Kaplunovsky and J. Louis, Nucl. Phys. B 422 (1994) 57 [arXiv:hep-th/9402005]. 